\title{
A Cost Utility Analysis of Prostate Cancer Screening in Australia
}

\author{
Dr. Andrew Keller ${ }^{1,2}$, Prof. Christian Gericke ${ }^{1,2}$, Assoc. Prof. Jennifer A Whitty ${ }^{1}$, Dr. John Yaxley ${ }^{3}$, \\ Dr. Boon Kua ${ }^{3}$, Dr Geoff Coughlin ${ }^{3}$ and Dr. TroyGianduzzo ${ }^{1,3}$.
}

\author{
Corresponding Author: Dr. Andrew Keller
}

Andrewthomaskeller@gmail.com

$+61438618610$

1. University of Queensland, Brisbane, QLD, Australia.

2. Wesley Research Institute, The Wesley Private Hospital, Brisbane, QLD Australia

3. The Wesley Private Hospital, Brisbane, QLD, Australia 


\section{$\underline{\text { Abstract }}$}

\section{Background and Objectives}

The Göteborg randomised population-based prostate cancer screening trial demonstrated that Prostate Specific Antigen (PSA) based screening reduces prostate cancer deaths compared with an age matched control group. Utilising the prostate cancer detection rates from this study we have investigated the clinical and costeffectiveness of a similar PSA-based screening strategy for an Australian population of men aged 50-69 years.

\section{Methods}

A decision model that incorporated Markov processes was developed from a health system perspective. The base case scenario compared a population-based screening programme with current opportunistic screening practices. Costs, utility values, treatment patterns and background mortality rates were derived from Australian data. All costs were adjusted to reflect July 2015 Australian dollars. An alternative scenario compared systematic with opportunistic screening but with optimisation of active surveillance (AS) uptake in both groups. A discount rate of 5\% for costs and benefits was utilised. Univariate and probabilistic sensitivity analyses were performed to assess the effect of variable uncertainty on model outcomes.

\section{Results}

Our model very closely replicated the number of deaths from bothprostate cancer and background mortality in the Göteborg study. The incremental cost per quality-adjusted life-year (QALY) for PSA screeningwas \$AU147,528. However, for years of life gained (LYGs) PSA based screening (\$AU45,890/LYG) appeared more favourable. Our alternative scenario with optimised AS improved cost-utility to \$AU45,881/QALY, with screening becoming cost-effective at a $92 \%$ AS uptake rate. Both modelled scenarios were most sensitive to the utility of patients before and after intervention, and the discount rate used.

\section{Conclusion}

PSA-based screening is not cost-effective compared to Australia's assumed willingness to pay threshold of \$AU50,000/QALY. It appears more cost-effective if LYGs are used as the relevant outcome, and is more cost effective than the established Australian breast cancer screening programme on this basis. Optimised utilisation of AS increases the cost-effectiveness of prostate cancer screeningdramatically.

Key Points for Decision Makers: 
- $\quad$ PSA-based prostate cancer screening is not cost effective in an Australianpopulation with current treatment patterns.

- Increased utilisation of active surveillance (AS) enhances the cost-effectiveness of prostatecancer screening, with screening becoming cost-effective at high AS uptake rates.

- At current Australian treatment patterns PC screening is more cost-effective than the current Australian breast cancer screening programme. 


\section{Introduction:}

Prostate cancer (PC) is currently the most commonly diagnosed cancer in Australia, afternon-melanomatous skin cancer, with Australia and New Zealand having amongst the highest incidences in the world.[1,2]

PC deaths in Australia totalled 3294 in 2011, the last year for which data is available, making itthe fourth most common cause of death in Australian males, and the second most common cause of cancer related death after lung cancer.[3] PC mortality consequently outnumbers both breast $(2,680)$ and sex specific bowel cancer deaths (2,219 deaths in men) both of which have established population based screening programmes.[3]

Despite the documented burden of disease that PC presents, the Royal Australian College of General Practioners' (RACGP) guidelines currently recommend against discussing the subject of prostate cancer screening unless the subject is raised by the patientthemselves.[4]

The RACGP cite the recent PSA based population screening studies of the Prostate, Lung, Colorectal and Ovarian Cancer Screening Trial (PLCO) and the 2009 results of the European Randomised Study of Screening for Prostate Cancer (ERSPC) as evidence of no mortality benefit for prostate specific antigen (PSA) based prostate cancer screening. [4] However, both PLCO and ERSPC studies suffered from methodological failures.[5] The PLCO suffered from a short screening period of only 6 years, a high proportion of randomised patients (44\%) having had a serum PSA performed in the last 3 years, and $52 \%$ of the control group receiving a PSA test over the 6 year screening period. [6] Compared to the Göteborg trial, the ERSPC suffered from heterogeneous, and on average higher, PSA thresholds $(2.5-10 \mathrm{ng} / \mathrm{mL})$ and heterogeneous, and on average longer, screening intervals (2-7 years) and shorter follow-up.[7,8]

The more recently published results of the Göteborg Randomised Population-based prostate-cancerscreening trial have demonstrated the greatest survival benefit and lowest number needed to treat (NNT) of any of the PSA screening trials.[8] Of the randomised controlled trials exploring PSA based population screening the Göteborg has the longest follow-up and the most robust trial protocol with a standardised serum PSA threshold (2.5ng/mL) and test interval of two years for the duration of the trial. [8]

The cost-effectiveness of prostate cancer screening has also been explored in numerous cost-effectiveness analyses (CEAs) which have produced widely varying results. No CEA to date, however, has used the findings of the Göteborg cohort, with most modelling the results of the 9 year follow-up data from the ERSPC, which had a significantly higher NNT.[9-11] 
Active surveillance (AS), an approach of monitoring rather than immediately treating low risk PC, has been demonstrated to decrease the harm of overtreatment, whilst preserving the benefits of screening.[12]Decision analyses investigating the cost-effectiveness of AS protocols compared to primary intervention for low risk prostate cancer found that AS dominated primary intervention at all modelled time horizons.[13, 14]

\section{Aim}

We aimed to investigate the predicted cost utility of a theoretical Australian PSA-based population screening programme. In our model, prostate cancer diagnoses were risk stratified, and any subsequent treatment based upon current Australian treatment patterns. The accumulated costs, utilities and cancer specific mortality rates between hypothetical screening and non-screening cohorts were recorded and compared. We aimed to compare two systematic screening strategies to current opportunistic screening practices: (i) a screening strategy where any cancer diagnoses would be treated in accordance with current Australian treatment patterns and (ii) an active surveillance-optimised strategy where anylow risk PC diagnoses were followed with an active surveillance protocol instead of receiving primary intervention until clinical disease progression.

\section{$\underline{\text { 2. Methodology }}$}

\subsection{Study Population}

The model evaluated the outcomes of a uniformly distributed Australian male cohorts aged between 50-69 years. A uniform distribution was selected to better reflect the recruitment method of the Göteborg study, as opposed to all men entering the model at a set age, as is commonly employed in other decision models.[9-11]

\subsection{Screening Strategies}

The model assessed the cost-utility of two screening strategies for prostate cancer: strategy 1) Asystematic population screening strategy, with invitation for screening with a serum PSA test every 2 years, with any suprathreshold PSA tests offered a Trans-Rectal Ultrasound guided biopsy (TRUS); strategy 2) Opportunistic prostate cancer screening, representing current standard practice. 
Two separate scenarios were created utilising the same two screening strategies. The first scenario, or base case,

compared both screening programmes with any subsequent prostate cancer diagnoses being treated in accordance with current Australian clinical practices, with treatment strategies determined by both cancer risk stratification and patient preference. The second scenario, or the AS-optimised model, differed inthat subsequent low risk prostate cancer diagnoses were all treated initially with AS instead of the majority of cases receiving primary intervention. Those on active surveillance that had PSA progression (PSA $\geq 10 \mathrm{ng} / \mathrm{mL})$ or PC upgrading (Gleason score >6) on subsequent TRUS biopsy would proceed to definitive intervention based upon their new risk stratification.

\subsection{Markov Model Structure and Health States}

To investigate the cost-utility of a PSA based screening programme in a simulated Australian population we built a decision model utilising Markov processes in TreeAge Pro 2014 software suite. (TreeAge Software Inc., Williamstown, MA, USA)

All men entered the model in a cancer-free/pre-diagnosis health state. Literature based probabilities sawmen either remain 1) disease free, 2) die of background (non-PC) causes or diagnosed with 3) low-risk, 4) intermediate-risk, 5) high-risk or 6) advanced (metastatic)PC.

Following diagnosis of non-metastatic PC the patient would either undergo AS (surveillance with curative intent, limited to those with low risk diagnoses), surgery, radiation or watchful waiting (WW; surveillance with palliative intent, limited to those with a life expectancy less than 10 years or contraindications to curative therapy). Curative treatment options were limited to the most commonly available Australian treatment options of Radical Retro-pubic Prostatectomy (RRP) and External Beam Radiation Therapy (EBRT). Following diagnosis and treatment men would stay in risk stratified post-treatment health states until natural deathor development of metastatic disease. (Figure 1)

In line with the Göteborg study, PSA screening would cease once a patient reached age 69 years, but they would continue to cycle through the model.[8] A one year cycle length was selected to best represent the slow natural history of PC. A 20 year time horizon was selected owing to the paucity of quality literature on both the natural history of PC and EBRT failure rates after this length of time. 


\subsubsection{Model Inputs}

Electronic literature searches utilising PubMed, Ovid MEDLINE and The Cochrane Library were performed to select model inputs for transition probabilities, utilities and costs. Australian studies were preferentially selected when available and where follow-up was appropriate for the model timeline. Where Australian studies were unable to wholly cover the timeline of the model, they were synthesized with estimates from international literature with longer follow-up. Where Australian studies were wholly lacking, mostly in the case oftransition probabilities, hand selection of the international literature was performed. Synthesized estimates were given preference over estimates from individual series. Where systematic reviews were non-existent, individualpapers were analysed, with those papers with protocols best representing current Australian practice, the largest numbers of recruited patients and the longest follow-up, where appropriate, being selected.

\subsubsection{Probabilities}

Probabilities of diagnoses of low, intermediate, high risk and advanced disease were drawn from the cumulative incidences for each diagnosis in both screening and control populations in the Göteborg study. [8] Yearly incidence rates were then calculated for each disease state diagnosis after adjusting for age and mortality related drop-out rates. Prostate cancer risk stratification in the Göteborg trial was based upon D’Amico’s 1998 classification for prostate cancer: Low risk - Gleason score on biopsy $\leq 6, \mathrm{PSA} \leq 10$, clinical stage $\leq \mathrm{T} 1 \mathrm{a}$, Intermediate risk - Gleason score $\leq 7, \mathrm{PSA} \leq 20$, clinical stage $\leq \mathrm{T} 2 \mathrm{~b}$, High risk - Gleason score $>7, \mathrm{PSA}>20$, clinical stage $>\mathrm{T} 2 \mathrm{~b}) .[15]$

Current treatment practices for each PC risk classification were drawn from Australian data.[16, 17]Treatments were limited to RRP, EBRT, WW and AS as these are the most universally available Australian treatment options. AS was only an option for patients diagnosed with low risk disease, consistent with currentEuropean Association of Urology guidelines and similar to those of the Prostate Cancer Foundation of Australia, which allows very low volume Gleason 3+4 and PSA values of $<20 \mathrm{ng} / \mathrm{mL} .[18,19]$ (Table 1) In the basecase (Scenario 1) the AS uptake rate was as per current Australian treatment patterns (15\% of low-risk diagnoses), in Scenario 2, all low risk diagnoses were treated with AS until clinical disease progression (serum PSA $\geq 10$, or Gleason score $>6$ on subsequent biopsy). 
Probability of progression to definitive therapy after entering the AS disease state was derived from a synthesis based estimate of AS series, and was dependant on time spent in the disease state.[20] Progression ratesinthe short-term (5 year) were similar to rates from an Australian AS cohort.[17]

A treatment threshold of equal or greater to 76 years old was selected as described in Campbell-WalshUrology. At this age all patients currently in the AS health state would transition to the WW health state.[21]

Probabilities of disease recurrence for each risk stratification following definitive treatment were derived from analysis of 6,652 D'Amico risk stratified men receiving PC therapy at a high volume centre.[22]

Probability of disease progression to metastasis for those in watchful waiting disease states was drawn from the Prostate Intervention Versus Observation Trial (PIVOT), being more contemporaneous (published 2012 vs 2002), with longer median follow-up (10 years vs 6.2) and having more participants in the observation arm(367 vs 349 men) than the next largest study published by Holmberg et al.[23,24]

Risk of progression to the advanced disease state following post-treatment recurrence was drawn froman international study which followed 2,426 men with biochemical recurrence after RRP with a median follow-up of 6.6 years.[25]

Adjustment for the possibility of initial clinical under-staging of low risk disease was performed, utilizing the results of the largest published prostatectomy specimen series of 626 patients meeting the strict ProstateCancer Research International: Active Surveillance (PRIAS) criteria.[26]

Probabilities of background mortality were drawn from Australian Bureau of Statistics age related mortality rates, and adjustments made for age related prostate cancer specific death rates.[27, 28] Although there is evidence androgen deprivation therapy (ADT) might increase the rate of cardiovascular events, an increase in cardiovascular mortality has not been conclusively demonstrated conclusively with significant discordance in results between published trials.[29-31] Additionally, in our model ADT was only initiated when metastases were present, reflecting more conservative practice and resulting in lower total ADT exposure. As such, we did not adjust the transition probability to non-cancer related death for those in the metastatic disease state. Wealso did not adjust for peri-operative mortality in the RRP group, however, Björklund et al's paper demonstrated that the 90 day per-operative mortality in $>22,000$ men receiving RRP was lower than the age-matched cohort $(<0.2 \%) \cdot[32]$ 


\subsubsection{Utility Values}

Health states were each assigned a utility value representing the health-related quality of life of anaverage patient inhabiting that disease state. All health state utility values were derived from Australian data where possible. (Table 2)

PC screening has been criticised as increasing anxiety and reducing health related quality of life (HRQoL) during and after diagnosis.[4] However, in the Dutch and Finnish centres of the ERSPC it was shown that screening does not induce short termHRQoL effect.[33, 34] Even those patients who had false positive results tended to regard screening as a positive experience.[35] Likewise participating in an active surveillance protocol does not appear to impact HRQoL or increase anxiety when compared to controls, with participants' HRQoL similar to age adjusted controls.[34, 36,37] Utility scores were consequently unaffected by screening or AS in our model.

We elected to use a single Australian study for our post-treatment utility scores. Smith et al. followed thesame large cohort (1500) of 50-69 year old men for 3 years before and after definitive treatment, comparing their utility directly against an age and pre-morbidity matched control cohort over the same three year period, using the same health instrument. This study utilised the University of California, prostate cancer index, a validated instrument which includes all 12 components of the commonly used 12-item short form (SF-12) questionnaire. They demonstrated a relative utility value of 0.95 when compared to age-matched controls at 3 yearsfollowing treatment.[36]

Despite widely ranging protocols for administration of androgen deprivation therapy our model conservatively initiated its usage only when patients transitioned to the metastatic health state. Of the treatment options androgen deprivation therapy is widely regarding as having the greatest effect on HRQoL, however, this may reflect the poor baseline of patients selected for androgen deprivation therapy.[36, 38] The metastatic health state utility value was drawn from a combination of Australian and international studies. [36, 39] Smith etal's Australian HRQoL study demonstrated a utility of 0.9 at initiation of androgen deprivation therapy, however follow-up was inadequate to assess the utility of terminal prostate cancer.[36] The utility of PC at its terminal stage was drawn from Farkilla et al, which assessed HRQoL using multiple health instruments (15D, EQ-5D, VAS, EORTC QLQ-C30) from which our utility value of terminal PC of 0.6 was synthesized.[39] The utilityof the advanced disease state thus fell from 0.9 to 0.6 over a five year period to represent deteriorating wellbeing 
with increasing burden of disease. This terminal illness health state utility is quite conservative compared to other cost utility analyses.[10, 38]

All utility values for disease-states listed above used above were not absolute values, but were multipliers for the age related baseline utility in a contemporary Australian male population.[40]

\subsubsection{Costs}

Costs were accumulated by both being in a specific disease state (state costs) as well discrete events (transition costs), such as having surgery.

A health system perspective was used to assign costs for screening, AS and treatment, as the model aims to approximate the costs of a government-run screening programme. Out of pocket and time costs forprogramme participants were therefore not included. Transition and state costings were derived from a combination of Australian studies, Medicare Benefits Schedule (MBS), Pharmaceutical Benefits Scheme (PBS) and Australian Refined - Diagnosis Related Groups (AR-DRGs). (Table 3) All costs were inflated to 10 July 2015 dollars using annual inflation rates from the Reserve Bank of Australia.

The cost of annual screening was calculated from the overall number of PSA blood tests and TRUS biopsies performed in the Göteborg study. A fractional annual test frequency per patient was calculated after adjusting for model dropout for either natural death or reaching the age limit of screening. The Australian costs of a serum PSA test and a TRUS biopsy, allowing for a $2 \%$ rate of post-TRUS sepsis were then used to arrive at thefinal cost of screening per man aged 50-69 in the screening cohort.[21]

\subsection{Model Outcomes}

The primary model output was Quality Adjusted Life Years (QALYs) for each screening strategyover 20 years.

Costs effectiveness was measured as an Incremental Cost Effectiveness Ratio (ICER) and expressed in \$/QALY. Life Years Gained (LYG) was used as an alternative model output to allow comparison with other cost-analyses that did not use the QALY metric. 
All future costs, QALYs and LYGs were discounted 5\% and compared to a societal willingness to pay (WTP) of \$50,000/QALY in line with Pharmaceutical Benefits Advisory Committee guidelines and a half-cyclecorrection was applied to all Markov processes. [41] Undiscounted QALYs and LYGs are also presented due to ongoing controversy regarding the discounting of future health outcomes, particularly for screening and prevention programmes.[42, 43] Cancer-specific mortalities, and interventions saved per 10,000 men was also used to compare treatment strategies.

\subsubsection{Univariant Sensitivity Analysis}

We conducted univariate sensitivity analyses of all probabilities, cost and utility values to determine the effect of uncertainty of each variable upon the models output. All cost and utility values were varied $20 \%$ above and below the base case value. Transition probabilities were varied within their $95 \%$ confidence intervals. Simulations were also run with a lifelong timeline and a cohort start age of 50 to assess possible impact on model outcomes.

Univariate sensitivity analysis outside of the confidence intervals was performed for the probability of entering the active surveillance health state following a diagnosis of low risk PC. This was done to determine what rate of primary active surveillance was required in order to achieve an increase in QALYs at a sub-thresholdWTP value.

\subsubsection{Probabilistic Sensitivity Analysis}

Multivariate probabilistic sensitivity analysis was performed for all variables in the both scenarios to assess overall model uncertainty. A second order Monte Carlo analysis was performed with all variables drawn simultaneously. Beta and gamma distributions were estimated for utility and costings values respectively from normal distributions with standard deviations of $20 \%$ above and below the base case values. A combination of Dirichlet and beta distributions were drawn for probability values based on their standard error values, as per Table 1. 10,000 samples were drawn for each probabilistic sensitivity analysis. 


\subsubsection{External validation}

In order to validate our models we ran our base case model with a 15 year time horizon and 9958 men in each arm, to simulate the follow-up and cohort numbers of the Göteborg. Our model very closely replicated the number of deaths from both prostate cancer and background mortality in the Göteborg study with 40 screening and 72 non-screening prostate cancer specific deaths, and 1791 screening and 1788 non-screening deathsfrom background mortality. This compares with 44 screening and 78 non-screening prostate cancer specificdeaths, and 1937 screening and 1904 non-screening deaths from background mortality in the Göteborg study.

\section{$\underline{3 \text { Results }}$}

\subsubsection{Scenario 1 - Base case}

With conservative modelling our theoretical population screening programme base case yielded an additional 0.00554 QALYs per patient at 20 years for an incremental cost of $\$ 817$ per patient when compared to the control group, opportunistic prostate cancer detection. This yielded an Incremental Cost Effectiveness Ratio (ICER) for screening of $\$ 147,528 / \mathrm{QALY}$.

With QALYs undiscounted an additional 0.01102 QALYs per patient were gained with the screening programme yielding an ICER of $\$ 74,165 /$ QALY.

With LYGs as the model output, screening yielded an additional 0.01781 LYGs per patient compared to current screening practices. This yielded an ICER of $\$ 45,890 / \mathrm{LYG}$. (Table 4)

\subsubsection{Scenario 2 - Optimised Active Surveillance}

In our active surveillance optimised scenario the screening programme generated higher incremental QALYs than Scenario 1 (base case), with an additional 0.01222 QALYs per patient when compared to theopportunistic screening group. The cost of screening was also lower than Scenario 1 , with an additional cost of $\$ 560$ per patient in the opportunistic screening group. This yielded an ICER for the AS-optimised screening scenario of $\$ 45,882 / \mathrm{QALY}$ compared to opportunistic screening. (Table 4)

With QALYs undiscounted an additional 0.0229 QALYS per patient were gained with the screening programme yielding an ICER of $\$ 24,483 /$ QALY. 
Whilst the AS-optimised screening Scenario 2 dominated Scenario 1(base case) in which usual treatment approaches were implemented, this increase in QALYs and reduction in cost compared to Scenario 1 came at the expense of one additional prostate cancer specific death per 10,000 men in both screened and unscreened cohorts. The number of interventions performed in both the screening (208 fewer RRP, 355 fewer EBRT) and non-screening groups per 10,000 men (105 fewer RRP, 149 fewer EBRT) was significantly reduced.

\subsubsection{Univariate Sensitivity Analyses}

Univariate sensitivity analyses of both modelled scenarios were dominated by utility following definitive therapy, particularly for Model 1. Varying the post-definitive therapy utility co-efficient from 0.95 to 0.90 led to net disutility for the entire screening cohort in Model 1, and resulted in the point estimate for Model 2 exceeding the WTP threshold. (Table 5)

After post-treatment utility the next most influential variables were, in order: age-related utility, discountrate and the utility of advanced disease. Cost variability within $20 \%$ of the base case value had little effect onoverall model output with almost all transition probabilities being more influential. Reducing the age of model entry to 50 years old, rather than utilising a uniformly distribution between ages 50-69, had the effect of reducingthe value of the point estimates without affecting the outcomes of the modelled scenarios. Similarly, utilising a lifelong timeline, as opposed to a 20 year time horizon resulted in lower point estimates without affecting scenario outcome. Whilst varying model inputs universally affected the value of the point estimates, it rarely affected the outcome; Scenario 1 remained cost in-effective and Scenario 2 remained cost-effective in most simulations. Results of the 4 variables with the most influence on ICER in the univariate sensitivity analysis are presented in Tornado diagrams (Figure 2 and 3) and the ICERs of the most influential variables are presented in Table 4.

When we varied the probability of entering the AS health state after a diagnosis of low-risk PC outside of its confidence intervals in Model 1 the screening arm became cost effective at an AS uptake rate of $91.9 \%$ assuming an acceptable threshold for cost-effectiveness of \$AU50,000/QALY. (Figure 4) 


\subsubsection{Probabilistic Sensitivity Analyses}

Probabilistic sensitivity analysis was performed for base model (Scenario 1) in order to ascertain the proportion of simulations in which it proved cost-effective. Assuming a willingness-to-pay threshold of \$AU50,000/QALY the base case model was cost effective in 38\% of simulations, delivered improved QALYs at supra-threshold WTP in 38\%, and decreased QALYs in $24.5 \%$ of simulations. (Figure 5)

For Scenario 2, Optimised Active Surveillance, the probabilistic sensitivity analysis demonstrated costeffectiveness in 53\% of all simulations, provided an increase in QALYs at a supra-threshold WTP in 27\% of simulations and decreased QALYs in 19\% of simulations. (Figure 6)

A cost-effectiveness acceptability curve was plotted for scenario 2 to demonstrate the effect of increasing WTP on the number of cost-effective iterations, at a WTP of \$100,000/QALY screening was cost effective in $66 \%$ of all iterations. (Figure 7)

\subsection{Discussion}

Our model is the first to explore the effectiveness and the cost of instituting a PSA-based populationscreening programme based on the results of the Göteborg Randomised Population-based prostate-cancer screening trial. Other CEAs to date have based their assumptions upon the results of the PLCO or ERSPC trials, however, the protocols of both trials were flawed.[5]

In our base case scenario, screening was not found to be cost effective as although it increased QALYs, it did so at an incremental cost of $\$ 147,528 / \mathrm{QALY}$, which was well above our nominal willingness to pay(WTP) of $\$ 50,000 /$ QALY.

When the results are viewed with a LYG metric the results appear more favourable, with a cost of $\$ 45,890 / \mathrm{LYG}$. It must be stressed that the seemingly large difference between the cost/QALY and cost/LYG is not due simply to patient disutility following treatment. Rather, as evidenced by the univariate sensitivity analysis, two of most influential model variables were the pre-morbid utility score and the discount rate of future outcomes/costs, consequently multiple lives needed to be extended in order to generate a single additional QALY. The cost-effectiveness of our model compares favourably with other CEAs, which have demonstrated highly variable results.[9] 
Pataky et al., in an ERSPC-based Canadian micro-simulation study, found screening strategies cost between

$\$ 27,000$ - \$54,000/LYG compared to non-screening strategies.[9] They also found that all strategies resulted in decreased QALYs for the screened population, however, this result was very sensitive to the utility values used.[9]

An American CEA based upon the NNT of the ERSPC (48 men) found at 9 years follow-up that screening was not cost-effective based upon their societal WTP of \$100,000/LYG.[11] However, they found that screening became cost effective when the NNT was less than 21, which compares favourably with the Göteborg's NNT of 12. $[8,11]$

Only one CEA is published that aims to model an Australian prostate cancer screening programme, and it is based upon the 9 year follow-up data from the ERSPC.[10] This model failed to demonstrate cost-utility fora population screening model in Australia, with a cost of \$291,817/QALY at 10 years follow-up, however, this analysis disregards the role of active surveillance completely.[10] Furthermore, every man in thismodel diagnosed with PC assumed a 0.05 disutility and all lifetime treatment costs immediately. This model also arguably overestimates costs by assuming the cost accrual of $100 \%$ screening compliance while assuming the compliance-unadjusted mortality reduction of the ERSPC which had only $82 \%$ of screened men having at least one PSA test, and $86 \%$ of men undergoing TRUS when recommended.[7]

Whilst our model suggests population-based screening at current AS uptake rates is not cost-effectivewhen compared to Australia's WTP, it does appear to compare favourably with established Australian screening programmes. BreastScreen Australia have not performed a comprehensive cost-utility analysis todirectly compare cost/QALY against.[44] They have, however, performed a CEA, with the Markov model output unit being cost per LYG. Similar to our model, BreastScreen Australia also focused on a population of 50-69 year olds, used a biennial screening test and a 5\% discount rate for future costs and benefits. After adjusting for inflation, the estimated cost per LYG over a 20 year time horizon was $\$ 47,776 / \mathrm{LYG}$.[44] Using the same LYG metric, a 5\% discount rate at a 20 year time horizon PSA based screening was more cost-effectiveat $\$ 45,890 /$ LYG.

Similarly, no true cost-utility analyses of the established Australian colon cancer screening programme have been performed, however, a CEA examining cost/LYG has been undertaken.[45] The projected cost of $\$ 53,989 / \mathrm{LYG}$, after adjustment to today's dollars, was higher than for our prostate cancer model, however, the time horizon the authors in this study selected was only 10 years. [46] 
A long-standing criticism of PC screening is the over-diagnosis and consequent overtreatment of clinically

indolent cancers. [7] The increasing uptake of active surveillance internationally might facilitate separation between low-grade PC diagnosis and its treatment.[47, 48] There is an increasing body ofevidence demonstrating that active surveillance can decrease the harm of overtreatment whilst maintaining the mortality benefits of screening.[12, 49] Indeed, while the base case for our model was found not to be cost-effectivebased upon current Australian treatment preferences, the cost/QALY wasshown to decrease dramatically with increased active surveillance utilisation. Other CEAs investigating the role of active surveillance in primary prostate cancer treatment have demonstrated that AS both increased QALYs and decreased treatment costs when compared to primary intervention.[13, 14] Koerber found that active surveillance dominated primary intervention for low risk PC, with AS yielding both lower costs and higher QALYs across modelled time horizons of 5, 15 and 30 years follow-up.[14] Orlendorf et al found that while AS yielded higherlifetime treatment costs than primary intervention this was offset by increased QALYs, with AS being the more costeffective treatment.[20] Our study extends these promising findings to suggest the AS-optimised model maybe cost-effective in the Australian setting.

The AS uptake rate required for cost effectiveness in our model was $92 \%$, which whilst high, may not be unattainable. Data from the Victorian Prostate Cancer Registry (VPCR) demonstrates increasing AS utilisation in recent years, with $36 \%$ of all new low risk diagnoses initially selecting AS.[50] In Sweden, AS is nowthe most commonly selected primary treatment for low risk PC, with $72 \%$ of all new diagnoses initially managed with AS.[51]

Concerns about clinical under-grading and under-staging of prostate cancer exist and are perhaps contributingto under-utilisation of AS as a treatment option for low risk prostate cancer.[26, 52] However, the clinical effect of an initial under-grading of prostate cancer in AS patients is yet to be established and it is not reason enough to deny patients the option of AS. The un-marrying of PC diagnosis and its immediate treatment and theincreased utilisation of AS as a treatment option are crucial to the cost-effectiveness of PSA based prostatecancer screening and essential in reducing screening-related harm.[53, 54]

This difficulty in establishing a disconnection between PC diagnosis and treatment might be alleviated by the increasing utilisation of multi-parametric Magnetic Resonance Imaging (mpMRI).[55]. MpMRI hasshown utility as a second line diagnostic tool in lieu of TRUS biopsy.[55] It allows preferential detection ofthose intermediate and high risk prostate cancers more likely to benefit from intervention, thereby avoiding detection 
of cancers unlikely to be clinically significant.[55] A cost-effectiveness analysis in the Netherlands has demonstrated similar costs for both mpMRI and TRUS pathways, but with a superior HRQoL in the mpMRI cohort due to reduced interventions for low risk disease.[56] In addition mpMRI has been demonstrated to aid correct risk stratification of patients prior to enrolment in an active surveillance protocol.[57]

\subsection{Limitations}

Our study has several limitations. Firstly probabilities are drawn from a prostate cancer screening study performed on a cohort of Swedish men. We did not adjust for Australia's higher incidence of PC as this may merely be an artefact of detection bias.[58]

While we did account for immediate costs of complications for RRP, such as readmission within 30 days for haematoma or infection, we did not account for cost of pads, incontinence surgeries or other treatment long-term complication costs. However, analysis of post-procedure costs in the sensitivity analysis reveals this ishighly unlikely to have had significant effect on the model's outcome.

Costs were not adjusted for societal costs of time off work for treatment, and likewise we did not adjust for loss of ability to work from terminal disease. However, it is argued by many health economists that these time costs exaggerate the true cost of a disease on the economy.[59] Our study was not intended to capture asocietal perspective; therefore, it may have underestimated the total societal costs associated with screening, treatment, or premature death.

A further limitation is utilisation of a time horizon of 20 years instead of lifelong time horizon. As demonstrated in our sensitivityanalysis, a longer time horizon led to increased cost effectiveness in both models, particularly

Scenario 1, although its ICER remained well above the WTP threshold, and as such did not change the decision outcome. (Table 4)

Another limitation is the use of non-time dependant probabilities for disease recurrence post-treatment, andfor development of metastases following disease recurrence. However, sensitivity analysis reveals the model to be relatively insensitive to these variables, and consequently it is unlikely thisaffected model outcomes.

The most significant limitation of our study compared to an actuarial population screening program is that the cohort of men that entered the model were aged between 50-69 in a uniform distribution to better parallel the recruitment method of the Göteborg study. A programme where all participants first underwent screening at age 
50 should allow earlier detection of prostate cancers and potentially increase the life years gained. When we ran our model with a uniform entry age of 50 years old, the cost-effectiveness of both models improved (Table 4). However, as the diagnostic probabilities were drawn from Göteborg data and not age-stratified these resultsare unlikely to be representative of the true benefit of commencing screening earlier.

Lastly, it should also be pointed out that the unscreened control cohort in the Göteborg study, whilst not formally invited to screening, were not PSA naïve, and still underwent a significant number of PSA tests and TRUS biopsies as a part of random opportunistic screening. In this regard, the Göteborg study, and consequently our model, represents the benefits of a formal population screening program compared to a population exposed to opportunistic screening only. In a truly unscreened, PSA naïve population, it is likely that the mortality differences would be greater still, and the cost per additional QALY considerably more favourable.

\section{Conclusions}

Our model is a comprehensive cost-utility analysis of a theoretical PSA-based prostate cancerscreening programme in Australia. In our base-case scenario prostate cancer population based screening with aninvitation to participate every 2 years for men aged 50-69 years was not found to be cost effective when compared to a commonly assumed willingness to pay threshold. It was, however, more cost effective per life year gained than the current Australian population screening programme for breast cancer. When the scenario optimised treatment post-diagnosis to focus on active surveillance, rather than primary intervention for low-riskdisease, our screening model was cost-effective after 20 years of follow-up. PSA based population screening maybe cost-effective when compared to opportunistic screening alone if low risk prostate cancer diagnoses can be successfully uncoupled from primary definitive treatment.

\section{Compliance with Ethical Standards}

\subsection{Research Funding}

This study was partially funded by a higher research scholarship from the Univeristy of Queensland (UQ). UQ did not have any influence on the subject matter of the study, model design or conclusions. No other external funding was received. 


\subsection{Conflicts of Interest}

The authors: Dr. Andrew Keller, Prof. Christian Gericke, Assoc. Prof. Jennifer Whitty, Dr. John Yaxley, Dr.

Boon Kua, Dr. Geoff Coughlin and Dr. Troy Gianduzzo have no conflicts of interest to declare.

\subsection{Ethics Approval}

Ethics approval was not required for this study.

\subsection{Author Contributions}

Dr. Andrew Keller created the models, performed the literature review and wrote the paper. Prof. Christain Gericke was involved in paper concept, model design and paper proofing. Assoc Prof. Jennifer Whitty was involved in model design and creation, proofing of paper and assistance with the health economic elements of the paper. Dr. John Yaxley, Dr. Boon Kua and Dr. GeoffCoughlin were involved in model design and proofing of the paper. Dr. TroyGianduzzo was responsible for the model and paper concept, was involved in model design and paper proofing and literature review.

\section{References}

1. Welfare AIoHa. Cancer in Australia: an overview 2012. Cancer series no 74 Cat no CAN 70 Canberra: AIHW [cited 13/06/2014] Available from:wwwaihwgovau.

2. Ferlay J SI, Ervik M, Dikshit R, Eser S, Mathers C, Rebelo M, Parkin DM, Forman D, Bray, F. GLOBOCAN 2012 v1.0, Cancer Incidence and MortalityWorldwide: IARC CancerBase No. 11 [Internet]. Lyon, France: International Agency for Research on Cancer; 2013 Available from: http://globocaniarcfr, [cited on $16 / 10 / 2014]$.

3. AIHW. Multiple causes of death in Australia: an analysis of all natural and selected chronic disease causes of death 1997-2007. AIHW bulletin no 105 Cat no AUS 159 Canberra: AIHW. 2012.

4. RACGP. Guidelines for preventive activities in general practice, 8th edn. East Melbourne: Royal Australian College of General Practitioners. 2012.

5. Eckersberger E FJ, Sadri H, , Margreiter M TS, Lepor H, Djavan B. Screening for Prostate Cancer: A Review of the ERSPC and PLCO Trials. Reviews in Urology.2009;11(3):127-33.

6. Andriole GL, Crawford ED, Grubb RL, 3rd, Buys SS, Chia D, Church TR, et al. Mortality results from a randomized prostate-cancer screening trial. The New England journal of medicine. 2009 Mar 26;360(13):1310-9.

7. Schröder F HJ, Roobol M, , Tammela T CSea. Screening and Prostate-Cancer Mortality in a Randomized European Study. NEJM. 2009;360(13):1320-8.

8. Hugosson J CS, Aus G, Bergdahl S, Khatami A, Lodding P, Pihl C, Stranne J, Holmberg E, L H. Mortality results from the Göteborg randomised population-based prostate-cancer screening trial. The lancet oncology. 2010;11:725-32.

9. Pataky R, Gulati R, Etzioni R, Black P, Chi KN, Coldman AJ, et al. Is prostate cancer screening costeffective? A microsimulation model of prostate-specific antigen-based screening for British Columbia, Canada. International journal of cancer Journal international du cancer. 2014 Jan 20.

10. Martin AJ, Lord SJ, Verry HE, Stockler MR, Emery JD. Risk assessment to guide prostate cancer screening decisions: a cost-effectiveness analysis. The Medical journal of Australia. 2013 Jun 3;198(10):546-50. 11. Shteynshlyuger A, Andriole GL. Cost-effectiveness of prostate specific antigen screening in the United States: extrapolating from the European study of screening for prostate cancer. The Journal of urology. 2011 Mar;185(3):828-32. 
12. Klotz L. Active surveillance for prostate cancer: patient selection and management. Curr Oncol. 2010

Sep;17 Suppl 2:S11-7.

13. Hayes JH, Ollendorf DA, Pearson SD, Barry MJ, Kantoff PW, Stewart ST, et al. Active surveillance compared with initial treatment for men with low-risk prostate cancer: a decision analysis. Jama. 2010 Dec $1 ; 304(21): 2373-80$.

14. Koerber F, Waidelich R, Stollenwerk B, Rogowski W. The cost-utility of open prostatectomy compared with active surveillance in early localised prostate cancer. BMC health services research. 2014;14(1):163.

15. D'Amico AV, Whittington R, Malkowicz SB, Schultz D, Blank K, Broderick GA, et al. Biochemical outcome after radical prostatectomy, external beam radiation therapy, or interstitial radiation therapy for clinically localized prostate cancer. Jama. 1998 Sep 16;280(11):969-74.

16. Baade PD, Youlden DR, Gardiner RA, Ferguson M, Aitken JF, Yaxley J, et al. Factors associatedwith treatment received by men diagnosed with prostate cancer in Queensland, Australia. BJU international. 2012 Dec;110(11 Pt B):E712-9.

17. Ischia JJ, Pang CY, Tay YK, Suen CF, Aw HC, Frydenberg M. Active surveillance for prostate cancer: an Australian experience. BJU international. 2012 Apr;109 Suppl3:40-3.

18. Prostate Cancer Foundation of Australia and Cancer Council Australia PSA Testing Guidelines Expert Advisory Panel. Draft clinical practice guidelines PSA Testing and Early Management of Test-DetectedProstate Cancer. [PDF on Internet] Sydney: Cancer Council Australia. [cited 2015 Jun 13]. Available from: http://wiki.cancer.org.au/australia/Guidelines:PSA_Testing/Active_surveillance.

19. Mottet N BJ, Briers E, van den Bergh R.C.N,, Bolla M vCNJ, Cornford P, Culine S JS, Lam T, Mason M.D, Matveev V, van der Poel H vdKTH, Rouvière O, Wiegel T. Guidelines on Prostate Cancer. European Association of Urology. 2015.

20. Orlendorf DA HJ, McMahon P, Pearson SD, . Active Surveillance and radical prostatectomy forthe management of low risk, clinically-localized prostate cancer - Final Appraisal Document. Institute for Clinical and Economic Review. 2009.

21. Wein AJ KL, Novick AC, Partin AW, Peters CA. Campbell-Walsh Urology 10th Edition: Elsevier Saunders; 2012.

22. Hernandez DJ, Nielsen ME, Han M, Partin AW. Contemporary evaluation of the D'amico risk classification of prostate cancer. Urology. 2007 Nov;70(5):931-5.

23. Holmberg L, Bill-Axelson A, Helgesen F, Salo JO, Folmerz P, Haggman M, et al. A randomized trial comparing radical prostatectomy with watchful waiting in early prostate cancer. The New England journal of medicine. 2002 Sep 12;347(11):781-9.

24. Wilt TJ, Brawer MK, Jones KM, Barry MJ, Aronson WJ, Fox S, et al. Radical prostatectomy versus observation for localized prostate cancer. The New England journal of medicine. 2012 Jul 19;367(3):203-13. 25. Boorjian SA, Thompson RH, Tollefson MK, Rangel LJ, Bergstralh EJ, Blute ML, et al. Long-termrisk of clinical progression after biochemical recurrence following radical prostatectomy: the impact of time from surgery to recurrence. European urology. 2011 Jun;59(6):893-9.

26. El Hajj A, Ploussard G, de la Taille A, Allory Y, Vordos D, Hoznek A, et al. Analysis of outcomes after radical prostatectomy in patients eligible for active surveillance (PRIAS). BJU international. 2013 Jan;111(1):53-9.

27. AIHW. Australian Institute of Health and Welfare (AIHW) 2014. Australian Cancer Incidence and Mortality (ACIM) books: Prostate cancer. Canberra: AIHW. [Cited 13/07/2015] Available from: http://www.aihw.gov.au/acim-books.; 2014.

28. Australian Bureau of Statistics DiAPoICCoA, [Cited 21 July 2015] Availabel from: http://www.ausstats.abs.gov.au/ausstats/subscriber.nsf/0/83A6580246688CEBCA2578840012A073/\$File/3303 0_2009.pdf. Deaths, Australia. 2009.

29. Carneiro A, Sasse AD, Wagner AA, Peixoto G, Kataguiri A, Neto AS, et al. Cardiovascular events associated with androgen deprivation therapy in patients with prostate cancer: a systematic review and metaanalysis. World journal of urology. 2014 Nov 12.

30. Jespersen CG, Norgaard M, Borre M. Androgen-deprivation therapy in treatment of prostatecancer and risk of myocardial infarction and stroke: a nationwide Danish population-based cohort study. European urology. 2014 Apr;65(4):704-9.

31. Voog JC, Paulus R, Shipley WU, Smith MR, McGowan DG, Jones CU, et al. Cardiovascular Mortality Following Short-term Androgen Deprivation in Clinically Localized Prostate Cancer: An Analysis of RTOG 9408. European urology. 2016 Feb;69(2):204-10.

32. Bjorklund J, Folkvaljon Y, Cole A, Carlsson S, Robinson D, Loeb S, et al. Postoperative mortality 90 days after robot-assisted laparoscopic prostatectomy and retropubic radical prostatectomy: a nationwide population-based study. BJU international. 2016 Jan 13. 
33. Essink-Bot ML, de Koning HJ, Nijs HG, Kirkels WJ, van der Maas PJ, Schroder FH. Short-term

effects of population-based screening for prostate cancer on health-related quality of life. Journal of the National Cancer Institute. 1998 Jun 17;90(12):925-31.

34. Vasarainen H, Malmi H, Maattanen L, Ruutu M, Tammela T, Taari K, et al. Effects of prostate cancer screening on health-related quality of life: results of the Finnish arm of the European randomized screening trial (ERSPC). Acta oncologica. 2013 Nov;52(8):1615-21.

35. Essink-Bot ML, Korfage IJ, De Koning HJ. Including the quality-of-life effects in the evaluationof prostate cancer screening: expert opinions revisited? BJU international. 2003 Dec;92 Suppl 2:101-5.

36. Smith DP, King MT, Egger S, Berry MP, Stricker PD, Cozzi P, et al. Quality of life three years after diagnosis of localised prostate cancer: population based cohort study. BMJ. 2009;339:b4817.

37. Wilcox CB, Gilbourd D, Louie-Johnsun M. Anxiety and health-related quality of life (HRQL) in patients undergoing active surveillance of prostate cancer in an Australian centre. BJU international. 2014 Mar;113 Suppl 2:64-8.

38. Cooperberg MR, Ramakrishna NR, Duff SB, Hughes KE, Sadownik S, Smith JA, et al. Primary treatments for clinically localised prostate cancer: a comprehensive lifetime cost-utility analysis. BJU international. 2013 Mar;111(3):437-50.

39. Farkkila N, Torvinen S, Roine RP, Sintonen H, Hanninen J, Taari K, et al. Health-related quality oflife among breast, prostate, and colorectal cancer patients with end-stage disease. Quality of life research : an international journal of quality of life aspects of treatment, care and rehabilitation. 2014 May;23(4):1387-94.

40. Banham D, Hawthorne G, Goldney R, Ratcliffe J. Health-Related Quality of Life (HRQoL) changes in South Australia: comparison of burden of disease morbidity and survey-based health utility estimates. Health and quality of life outcomes. 2014 Aug 5;12(1):113.

41. Pharmaceutical Benefits Advisory Committee. Guidelines for preparing submissions to the

Pharmaceutical Benefits Advisory Committee (Version 4.5). Canberra: DoHA, January 2014.

42. Severens JL, Milne RJ. Discounting health outcomes in economic evaluation: the ongoing debate. Value Health. 2004 Jul-Aug;7(4):397-401.

43. Oliver A. A normative perspective on discounting health outcomes. J Health Serv Res Policy. 2013 Jul;18(3):186-9.

44. Australia B. BreastScreen Australia Evaluation - Evaulation Final Report.2009.

45. O'Leary BA, Olynyk JK, Neville AM, Platell CF. Cost-effectiveness of colorectal cancer screening: comparison of community-based flexible sigmoidoscopy with fecal occult blood testing and colonoscopy. Journal of gastroenterology and hepatology. 2004 Jan;19(1):38-47.

46. Albert M, Tempany CM, Schultz D, Chen MH, Cormack RA, Kumar S, et al. Late genitourinary and gastrointestinal toxicity after magnetic resonance image-guided prostate brachytherapy with or without neoadjuvant external beam radiation therapy. Cancer. 2003 Sep 1;98(5):949-54.

47. Loeb S, Bjurlin MA, Nicholson J, Tammela TL, Penson DF, Carter HB, et al. Overdiagnosis and overtreatment of prostate cancer. European urology. 2014 Jun;65(6):1046-55.

48. Murphy DG, Loeb S. Prostate cancer: Growth of AS in the USA signals reduction in overtreatment. Nature reviews Urology. 2015 Sep 22.

49. Godtman RA, Holmberg E, Khatami A, Stranne J, Hugosson J. Outcome following active surveillance of men with screen-detected prostate cancer. Results from the Göteborg randomised population-based prostate cancer screening trial. European urology. 2013 Jan;63(1):101-7.

50. Weerakoon M, Papa N, Lawrentschuk N, Evans S, Millar J, Frydenberg M, et al. The current useof active surveillance in an Australian cohort of men: a pattern of care analysis from the Victorian Prostate Cancer Registry. BJU international. 2015 Apr;115 Suppl 5:50-6.

51. Loeb S, Folkvaljon Y, Makarov DV, Bratt O, Bill-Axelson A, Stattin P. Five-year nationwide followup study of active surveillance for prostate cancer. European urology. 2015 Feb;67(2):233-8.

52. Oliveira IS, Pontes-Junior J, Abe DK, Crippa A, Dall'oglio MF, Nesralah AJ, et al. Undergrading and understaging in patients with clinically insignificant prostate cancer who underwent radical prostatectomy. International braz j urol : official journal of the Brazilian Society of Urology. 2010 May-Jun;36(3):292-9. 53. Sartor O. Expert Editorial: The PSA Screening Conundrum Revisited [Cited 17/10/2014] Available from: http://gucasym.org/expert-editorial-psa-screening-conundrum-revisited.

54. Murphy DG, Ahlering T, Catalona WJ, Crowe H, Crowe J, Clarke N, et al. The Melbourne Consensus Statement on the early detection of prostate cancer. BJU international. 2014 Feb;113(2):186-8.

55. Pokorny MR, de Rooij M, Duncan E, Schroder FH, Parkinson R, Barentsz JO, et al. Prospective Study of Diagnostic Accuracy Comparing Prostate Cancer Detection by Transrectal Ultrasound-Guided Biopsy Versus Magnetic Resonance (MR) Imaging with Subsequent MR-guided Biopsy in Men Without Previous Prostate Biopsies. European urology. 2014 Jul;66(1):22-9. 
56. de Rooij M, Crienen S, Witjes JA, Barentsz JO, Rovers MM, Grutters JP. Cost-effectiveness of Magnetic Resonance (MR) Imaging and MR-guided Targeted Biopsy Versus Systematic Transrectal Ultrasound-Guided Biopsy in Diagnosing Prostate Cancer: A Modelling Study from a Health Care Perspective. European urology. 2013 Dec 21.

57. Turkbey B, Mani H, Aras O, Ho J, Hoang A, Rastinehad AR, et al. Prostate cancer: can multiparametric MR imaging help identify patients who are candidates for active surveillance? Radiology. 2013 Jul;268(1):144-52.

58. Ferlay J, Soerjomataram I, Dikshit R, Eser S, Mathers C, Rebelo M, et al. Cancer incidence and mortality worldwide: Sources, methods and major patterns in GLOBOCAN 2012. Int J Cancer. 2014 Sep 13. 59. Drummond MF SM, Torrance, GW, O'Brien BJ, Stoddart GL. Methods for the Economic Evaluation of Health Care Programmes 3rd Edition: Oxford University Press; 2005.

60. Baade PD, Aitken JF, Ferguson M, Gardiner RA, Chambers SK. Diagnostic and treatment pathways for men with prostate cancer in Queensland: investigating spatial and demographic inequalities. BMC Cancer. 2010;10:452.

61. Maximum androgen blockade in advanced prostate cancer: an overview of the randomised trials. Prostate Cancer Trialists' Collaborative Group. Lancet. 2000 Apr 29;355(9214):1491-8.

62. Hall RM, Linklater N, Coughlin G. Robotic and open radical prostatectomy in the public health sector: cost comparison. ANZ journal of surgery. 2014 Jun;84(6):477-80.

63. Baade PD, Gardiner RA, Ferguson M, Youlden DR, Aitken JF, Yaxley J, et al. Factors associatedwith diagnostic and treatment intervals for prostate cancer in Queensland, Australia: a large cohort study. Cancer Causes Control. 2012 Apr;23(4):625-34.

64. Yaxley J, Yaxley J, Gardiner R, Yaxley W. Prostate cancer - active surveillance as amanagement option. Australian family physician. 2013 Jan-Feb;42(1-2):74-6.

65. Carter HE, Martin A, Schofield D, Duchesne G, Haworth A, Hornby C, et al. A decision model to estimate the cost-effectiveness of intensity modulated radiation therapy (IMRT) compared to three dimensional conformal radiation therapy (3DCRT) in patients receiving radiotherapy to the prostate bed. Radiotherapy and oncology : journal of the European Society for Therapeutic Radiology and Oncology. 2014 Jun 11.

66. Hollander MJ. Costs of end-of-life care: findings from the province of Saskatchewan. Healthcare quarterly. 2009;12(3):50-8.

67. Krahn MD, Zagorski B, Laporte A, Alibhai SM, Bremner KE, Tomlinson G, et al. Healthcare costs associated with prostate cancer: estimates from a population-based study. BJU international. 2010

Feb;105(3):338-46. 


\section{Legends To Figures}

Figure 1. Simplified state transition diagram demonstrating possible cancer diagnoses and treatment decisions that may be made, and subsequent disease states that may be entered by each simulated patient passing through the prostate cancer disease model.

Figure 2. Incremental Cost Effectiveness Ratio (ICER) Tornado diagram of univariate sensitivity analysis of all costs, probabilities and utility values in base case simulation (Scenario 1). The 4 most influential model variables are presented. ICER is represented in Australia dollars per quality adjusted life year gained (\$/QALY).

Figure 3. Incremental Cost Effectiveness Ratio (ICER) Tornado diagram of univariate sensitivity analysis ofall costs, probabilities and utility values in alternative model (Scenario 2). The 4 most influential model variables are presented. ICER is represented in Australia dollars per quality adjusted life year gained (\$/QALY).

Figure 4. Primary Active Surveillance Rate for New Diagnoses of Low Risk Prostate Cancer versus Net Monetary Benefits $(\mathrm{NMB}=$ expected QALYs x WTP - expected cost). Discount Rate 5\%, Willingness To Pay (WTP) of $\$ 50,000$, modelled time horizon of 20 years. The screening model becomes cost effective at an active surveillance uptake of $91.9 \%$.

Figure 5. Probabilistic Sensitivity Analysis Scatter Plot, Base Case Scenario (Scenario 1). Output in Incremental Cost Effectiveness, Screening Vs. No-Screening.

Figure 6. Probabilistic Sensitivity Analysis Scatter Plot, Active Surveillance Optimised (Scenario 2) Output in Incremental Cost Effectiveness, Screening Vs.No-Screening.

Figure 7. Cost-Effectiveness Acceptability Curve, Active Surveillance Optimised (Scenario 2). Number of costeffective iterations versus societal Willingness To Pay (WTP). WTP varied between 0-\$100,000 Australian dollars per quality adjusted life year gained. (\$AUD/QALY). 


\section{Tables}

\subsection{Table 1 Health State Transition Probabilities}

\begin{tabular}{|c|c|c|c|c|}
\hline State & Event & $\begin{array}{l}\text { Transition } \\
\text { probability }\end{array}$ & Standard Error & Source \\
\hline \multirow{4}{*}{ Screening } & Diagnosed Low Risk CaP & 0.0045824791 & 0.0006770141 & Hugosson, J. 2010[8] \\
\hline & Diagnosed Intermediate Risk CaP & 0.0027211009 & 0.0005221863 & Hugosson, J. 2010[8] \\
\hline & Diagnosed High Risk CaP & 0.0007103095 & $2.67 \mathrm{E}-04$ & Hugosson, J. 2010[8] \\
\hline & Diagnosed Advanced CaP & 0.000339538 & $1.85 \mathrm{E}-04$ & Hugosson, J. 2010[8] \\
\hline \multirow{4}{*}{ Non-Screening } & Diagnosed Low Risk CaP & 0.001567317 & 0.000396536 & Hugosson, J. 2010[8] \\
\hline & Diagnosed Intermediate Risk CaP & 0.0019661929 & 0.0004440486 & Hugosson, J. 2010[8] \\
\hline & Diagnosed High Risk CaP & 0.0009886531 & $3.15 \mathrm{E}-04$ & Hugosson, J. 2010[8] \\
\hline & Diagnosed Advanced CaP & 0.0006812806 & $2.62 \mathrm{E}-04$ & Hugosson, J. 2010[8] \\
\hline \multirow{4}{*}{$\begin{array}{l}\text { Diagnosed Low Risk } \\
\text { CaP }\end{array}$} & Proceed to EBRT & 0.472 & 0.0352998584 & Baade PD, 2012[60] \\
\hline & Proceed to RRP & 0.321 & 0.0330120433 & Baade PD, 2012[60] \\
\hline & Proceed to WW & 0.057 & 0.0163937488 & Baade PD, 2012[60] \\
\hline & Proceed to AS & \# & & \\
\hline \multirow{3}{*}{$\begin{array}{l}\text { Diagnosed } \\
\text { Intermediate Risk } \\
\text { CaP }\end{array}$} & Proceed to EBRT & 0.4008127208 & 0.0205988903 & Baade PD, 2012[60] \\
\hline & Proceed to RRP & 0.534 & 0.020967921 & Baade PD, 2012[60] \\
\hline & Proceed to WW & \# & & \\
\hline \multirow{3}{*}{$\begin{array}{l}\text { Diagnosed High Risk } \\
\text { CaP }\end{array}$} & Proceed to EBRT & 0.4513333333 & 0.0374038546 & Baade PD, 2012[60] \\
\hline & Proceed to RRP & 0.452 & 0.0374087213 & Baade PD, 2012[60] \\
\hline & Proceed to $\mathrm{WW}$ & \# & & \\
\hline \multirow{4}{*}{$\begin{array}{l}\text { Active Surveillance } \\
\text { <76 years }\end{array}$} & $\begin{array}{l}\text { Develop Advanced Disease } \\
\text { whilst on AS }\end{array}$ & 0.00523 & 0.000425 & Koerber F 2014[14] \\
\hline & $\begin{array}{l}\text { Proceed to definitive treatment } \\
1 \text { st } 5 \text { years }\end{array}$ & 0.0643706623 & 0.0077606108 & Orlendorf DA 2009[20] \\
\hline & $\begin{array}{l}\text { Proceed to definitive treatment } \\
\text { 2nd } 5 \text { years }\end{array}$ & 0.0323158832 & 0.0055920986 & Orlendorf DA 2009[20] \\
\hline & $\begin{array}{l}\text { Proceed to definitive treatment } \\
\text { 3rd } 5 \text { years }\end{array}$ & 0.0188410491 & 0.0042995423 & Orlendorf DA 2009[20] \\
\hline $\begin{array}{l}\text { Active Surveillance } \\
\geq 76 \text { years }\end{array}$ & Proceed to WW & 1 & N/A & $\begin{array}{l}\text { Campbell-Walsh } \\
\text { Urology[21] }\end{array}$ \\
\hline \multirow{3}{*}{ AS To Definitive } & Proceed to EBRT & 0.2962962963 & 0.0367957508 & Ischia JJ, 2012[17] \\
\hline & Proceed to RRP & 0.6296296296 & 0.0389135037 & Ischia JJ, 2012[17] \\
\hline & Proceed to WW & \# & & \\
\hline
\end{tabular}




\begin{tabular}{|c|c|c|c|c|}
\hline Clinical & Upstaged to Intermediate Risk & 0.294 & 0.0182091154 & El Hajj, A. 2013[26] \\
\hline $\begin{array}{l}\text { Understaging of Low } \\
\text { Risk Disease }\end{array}$ & Upstaged to High Risk & 0.206 & 0.0161642923 & El Hajj, A. 2013[26] \\
\hline $\begin{array}{l}\text { Post Treatment Low } \\
\text { Risk }\end{array}$ & Recurrence & 0.0112503069 & 0.001293151 & Hernandez DJ, 2007[22] \\
\hline $\begin{array}{l}\text { Post Treatment } \\
\text { Intermediate Risk }\end{array}$ & Recurrence & 0.0797437258 & 0.0033214409 & Hernandez DJ, 2007[22] \\
\hline $\begin{array}{l}\text { Post Treatment High } \\
\text { Risk }\end{array}$ & Recurrence & 0.1139901939 & 0.0038965198 & Hernandez DJ, 2007[22] \\
\hline $\begin{array}{l}\text { Post Treatment } \\
\text { Recurrence }\end{array}$ & Progress To Advanced Disease & 0.0127 & 0.0016097136 & Boorjian SA, 2011[25] \\
\hline $\begin{array}{l}\text { WW Low Risk } \\
\text { Disease }\end{array}$ & Progress To Advanced Disease & 0.0062604731 & 0.0041172461 & Wilt TJ, 2012[24] \\
\hline $\begin{array}{l}\text { WW Intermediate } \\
\text { Risk Disease }\end{array}$ & Progress To Advanced Disease & 0.0151549445 & 0.0063771715 & Wilt TJ, 2012[24] \\
\hline $\begin{array}{l}\text { WW High Risk } \\
\text { Disease }\end{array}$ & Progress To Advanced Disease & 0.024055818 & 0.0079981496 & Wilt TJ, 2012[24] \\
\hline Advanced Disease & Die of Advanced $\mathrm{CaP}$ & 0.224 & 0.0045832217 & PCTCG. 2000[61] \\
\hline ALL GROUPS & Die Background Mortality & Age dependant & \multicolumn{2}{|c|}{$\begin{array}{l}\text { ABS Mortality rates, adjusted for age related } \\
\text { prostate cancer mortality }\end{array}$} \\
\hline
\end{tabular}

$\#=$ Sums to 1.0 with other probabilities in health state.

$\mathrm{CaP}=$ Prostate Cancer

$\mathrm{RRP}=$ Retro-pubic radical prostatectomy

EBRT $=$ External Beam Radiation Therapy

$\mathrm{WW}=\mathrm{Watchful}$ waiting

$\mathrm{AS}=$ Active Surveillance 
9.2 Table 2 Health State Utility Values

\begin{tabular}{|c|c|c|}
\hline State & Utility & Source \\
\hline Baseline utility value & Age dependant & Banham D. 2014[40] \\
\hline Screening* & 1 & Vasarainen H. 2013[34] \\
\hline Non-Screening* & 1 & Smith DP. 2009[36] \\
\hline Post Treatment* & 0.95 & Smith DP. 2009[36], Farkkila N. \\
\hline Advanced Disease* & $0.9->0.6$ over 5 years & 2014[39] \\
\hline
\end{tabular}

$*=$ Values are not absolute, but multipliers of the age dependant baseline utility value. 
9.3 Table 3 Health State and Transition Costs

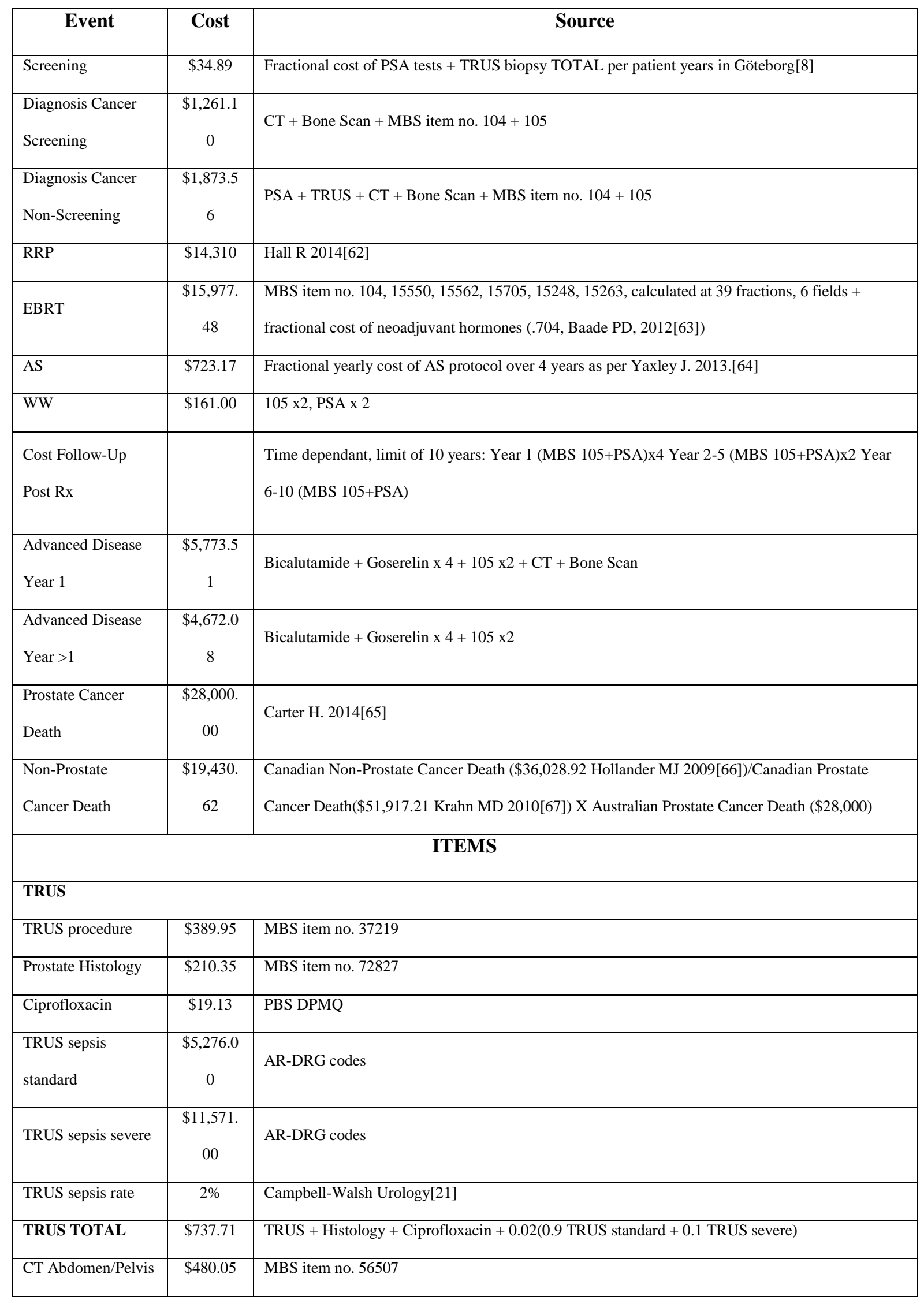




\begin{tabular}{|c|c|c|}
\hline Tc99m Bone Scan & $\$ 489.70$ & MBS item no. 61441 \\
\hline $\begin{array}{l}\text { Initital specialist } \\
\text { consultation }\end{array}$ & $\$ 85.55$ & MBS item no. 104 \\
\hline $\begin{array}{l}\text { Review specialist } \\
\text { consultation }\end{array}$ & $\$ 43.00$ & MBS item no. 105 \\
\hline PSA & $\$ 37.55$ & MBS item no. 66660 \\
\hline \multicolumn{3}{|c|}{ Androgen Deprivation Therapy (ADT) } \\
\hline Bicalutamide & $\$ 131.68$ & PBS DPMQ \\
\hline Goserelin & $\begin{array}{c}\$ 1,108.9 \\
7\end{array}$ & PBS DPMQ \\
\hline $\begin{array}{l}\text { Neoadjuvant } \\
\text { hormones }\end{array}$ & $\$ 2349.62$ & Bicalutamide $\mathrm{x} 1+$ Goserelin $\mathrm{x} 2$ \\
\hline
\end{tabular}

$\mathrm{CaP}=$ Prostate Cancer

$\mathrm{RRP}=$ Retro-pubic radical prostatectomy

EBRT $=$ External Beam Radiation Therapy

$\mathrm{WW}=$ Watchful waiting

AS = Active Surveillance

$\mathrm{CT}=$ Computerised Tomography

TRUS = Trans-Rectal Ultrasound guided prostate biopsy 
9.4 Table 4: Results

\begin{tabular}{|c|c|c|c|c|c|}
\hline & Cost $(\$)$ & QALYs & LYG & $\begin{array}{l}\text { Cancer } \\
\text { deaths/10,000 } \\
\text { men }\end{array}$ & $\begin{array}{l}\text { Non-Cancer } \\
\text { deaths/10000 men }\end{array}$ \\
\hline MODEL 1: Basecase & \multicolumn{5}{|c|}{ (20 years, $5 \%$ discount for both cost and utility) } \\
\hline Control & $\$ 4,664.17$ & 9.32618 & 11.58002 & 98 & 2809 \\
\hline Screening & $\$ 5,481.47$ & 9.33172 & 11.59783 & 59 & 2816 \\
\hline Incremental & $\$ 817.30$ & 0.00554 & $\mathbf{0 . 0 1 7 8 1}$ & -39 & 7 \\
\hline ICER & \multicolumn{2}{|c|}{ \$147,528/QALY } & \multicolumn{2}{|c|}{$\$ 45,890 / L Y G$} & \\
\hline MODEL 2: Optimised AS & \multicolumn{5}{|c|}{ (20 years, $5 \%$ discount for both cost and utility) } \\
\hline Control & $\$ 4,524.92$ & 9.32974 & 11.57838 & 99 & 2809 \\
\hline Screening & $\$ 5,085.59$ & 9.34196 & 11.59619 & 60 & 2816 \\
\hline Incremental & $\$ 560.67$ & 0.01222 & $\mathbf{0 . 0 1 7 8 1}$ & -39 & 7 \\
\hline ICER & \multicolumn{2}{|c|}{$\$ 45,882 / Q A L Y$} & \multicolumn{2}{|c|}{$\$ 31,840 / L Y G$} & \\
\hline MODEL 1: Basecase & \multicolumn{5}{|c|}{ No discount on utilities. $5 \%$ discount on costs. } \\
\hline Control & $\$ 4,664.17$ & $\mathbf{1 3 . 7 8 5 8 5}$ & $\mathbf{1 7 . 6 4 4 5}$ & 98 & 2809 \\
\hline Screening & $\$ 5,481.47$ & 13.79692 & 17.6788 & 59 & 2816 \\
\hline Incremental & $\$ 817.30$ & 0.01107 & $\mathbf{0 . 0 3 4 3}$ & -39 & 7 \\
\hline ICER & \multicolumn{2}{|c|}{ \$103,965/QALY } & \multicolumn{2}{|c|}{$\$ 23,828 / L Y G$} & \\
\hline MODEL 2: Optimised AS & \multicolumn{5}{|c|}{ No discount on utilities. $5 \%$ discount on costs. } \\
\hline Control & $\$ 4,524.92$ & 13.79227 & 17.6446 & 99 & 2809 \\
\hline Screening & $\$ 5,085.59$ & 13.81517 & 17.67908 & 60 & 2816 \\
\hline Incremental & $\$ 560.67$ & 0.0229 & 0.03448 & -39 & 7 \\
\hline ICER & \multicolumn{2}{|c|}{ \$24,483.41/QALY } & \multicolumn{2}{|c|}{$\$ 16,261 / L Y G$} & \\
\hline
\end{tabular}


9.5 Table 5: Sensitivity Analysis

\begin{tabular}{|l|l|l|}
\hline Variables & \multicolumn{2}{|c|}{ New ICER } \\
\hline Discount Rates & MODEL 1 & MODEL 2 \\
\hline $2 \%$ Discount for both & $\$ 122,711 /$ QALY & $\$ 40,193 /$ QALY \\
\hline $8 \%$ Discount for both & $\$ 177,932 /$ QALY & $\$ 50,664 /$ QALY \\
\hline Utility & & $\$ 33,021 /$ QALY \\
\hline Starting Utility 1.0 (Rather than age related) & $\$ 103,957 /$ QALY & $\$ 58,010 /$ QALY \\
\hline Utility Post- Treatment 0.9 & Net dis-utility for screening & \\
\hline Timeline & cohort & \\
\hline Lifelong timeline & & \\
\hline Age of model entry & $\$ 103,565 /$ QALY & \\
\hline All patients start screening at 50 & & \\
\hline
\end{tabular}




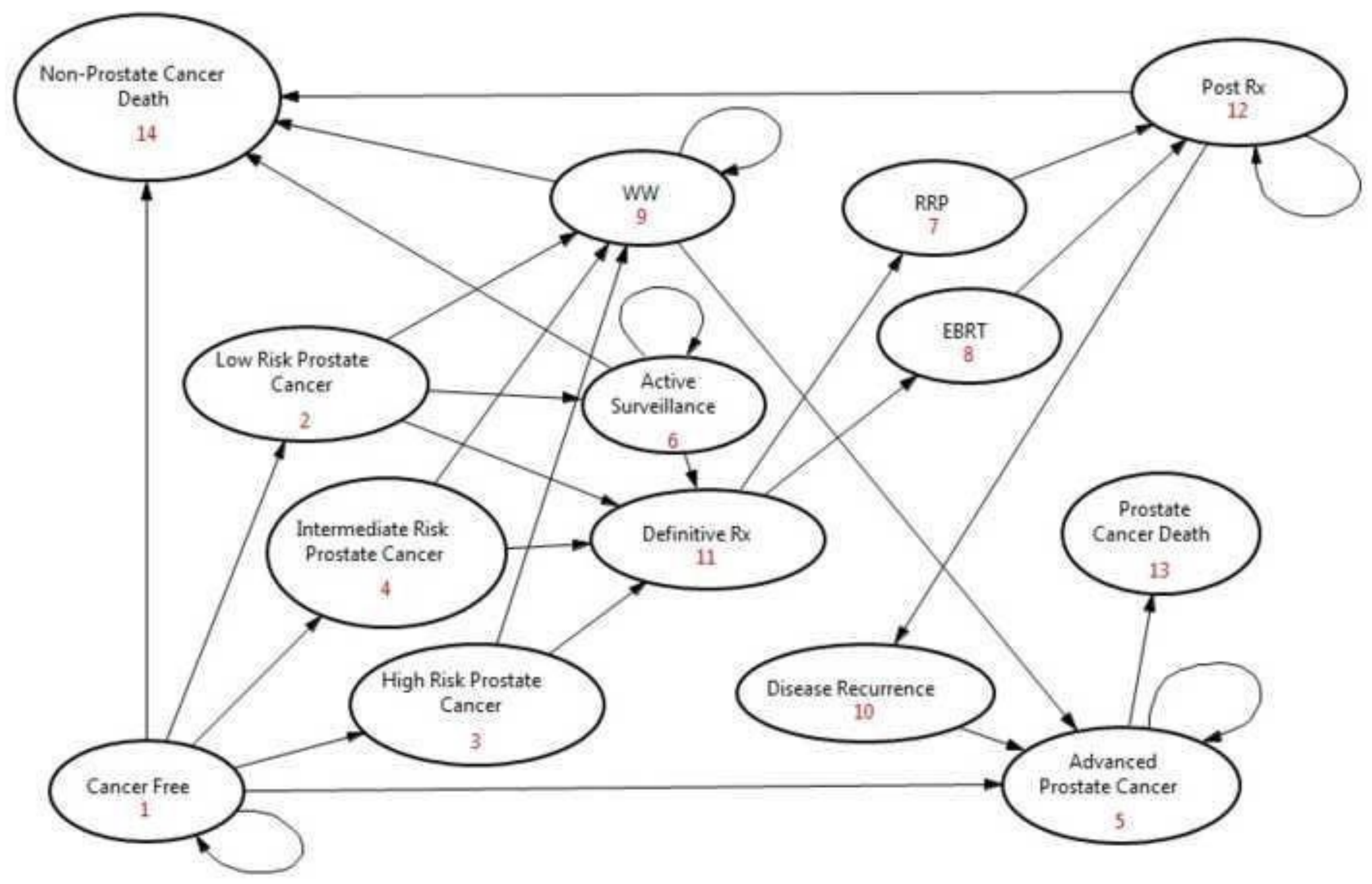




\section{Tornado Analysis (ICER)}

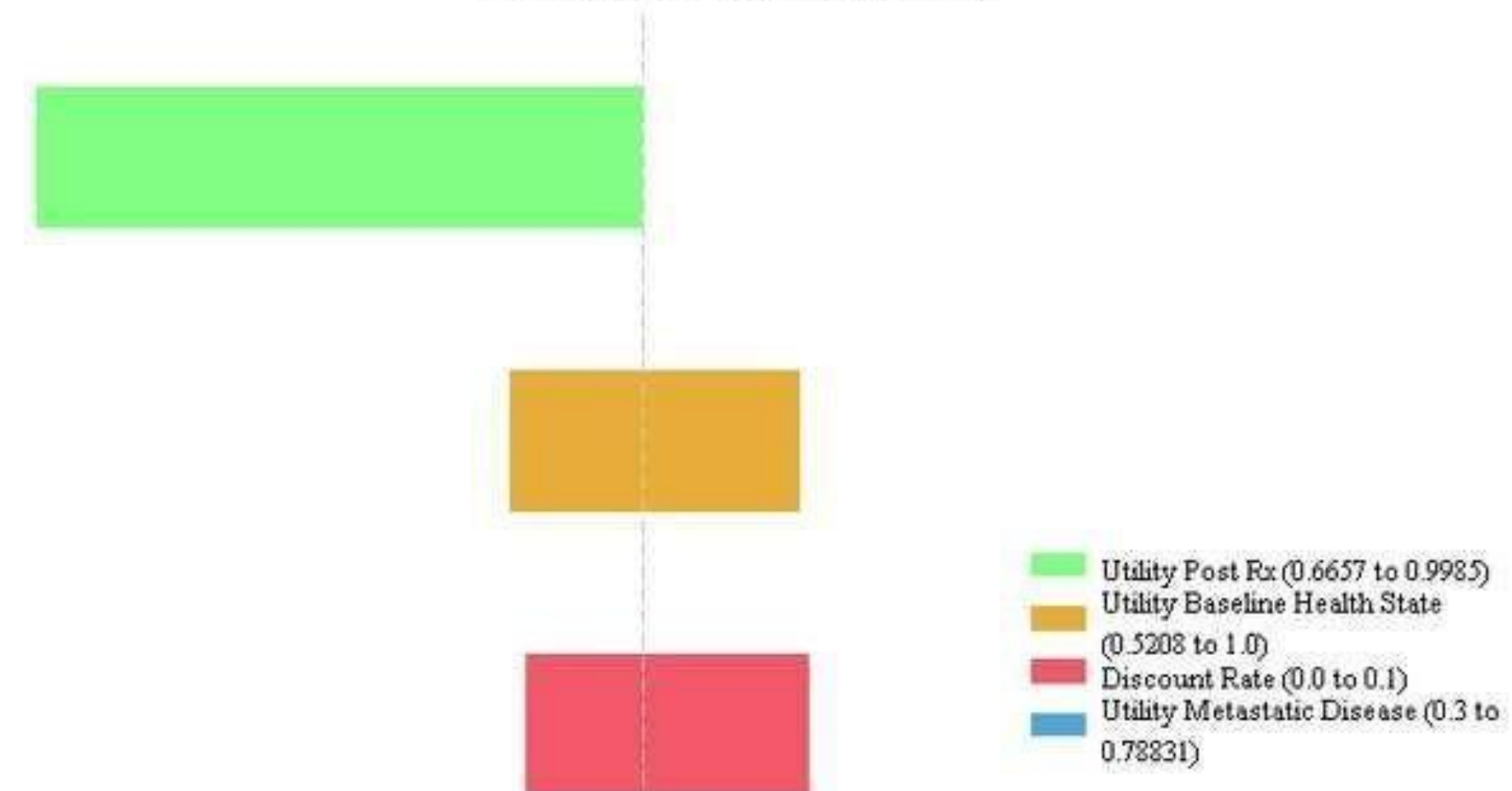

EV : 148127.40522

$\begin{array}{cccccc}-100000 & -50000 & 0 & 50000 \quad 100000 \quad 150000 & 200000 & 250000 \\ & & \\ \text { ICER/SAUD/QALY) }\end{array}$




\section{Tornado Analysis (ICER)}

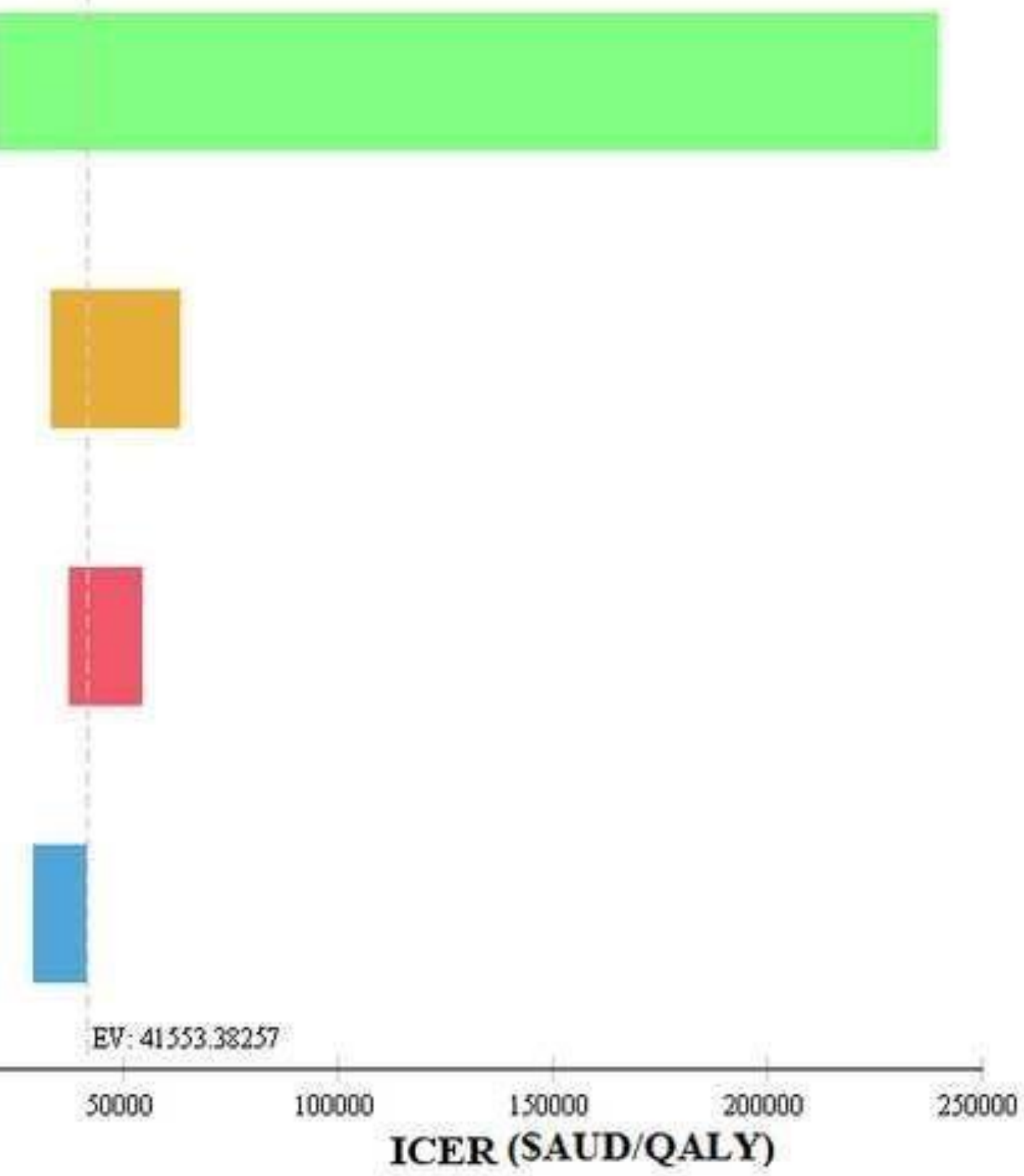

Utsility Post $\mathrm{R} \times(0.6657$ to 0.9985$)$

Utility Baseline He alth State

(0.5208 to 1.09

Discount Rate $(0.0$ to 0.1$)$

Utsily Metastatic Dise ase (0.3 to

0.78831 )

$\begin{array}{rrrr}50000 & 100000 & 150000 & 200000\end{array}$


Your image file "Figure 4 Univariate NMB 5\%.jpg" cannot be opened and processed. Please see the common list of problems, and suggested resolutions below.

Reason: The image file is corrupt or invalid. Please check and resubmit.

Other Common Problems When Creating a PDF from an image file

You will need to convert your image file to another format or fix the current image, then re-submit it. 


\section{Probabilistic Sensitivity Analysis - Incremental Cost-Effectiveness, Screening v. No Screening}

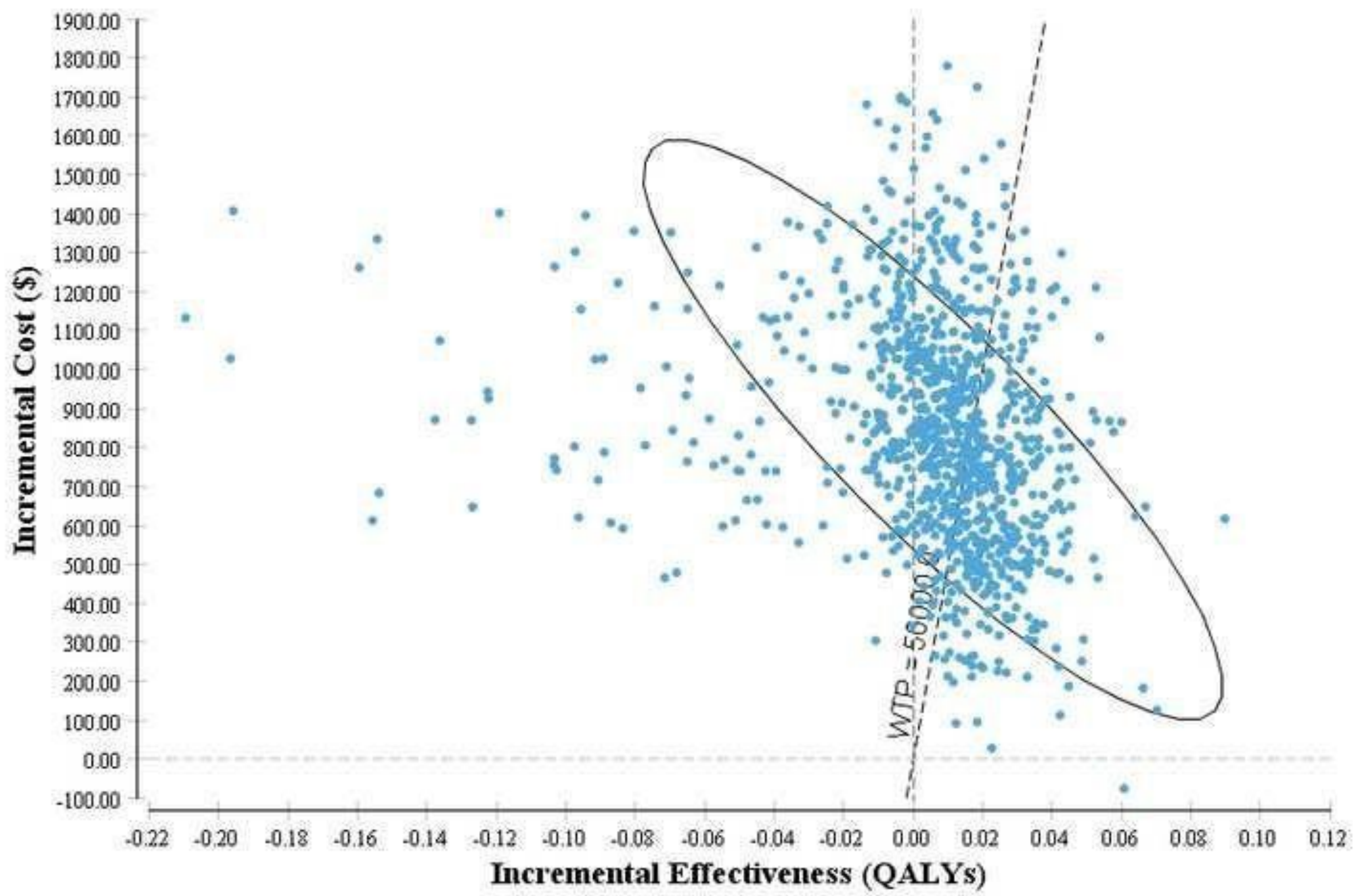


Probabilistic Sensitivity Analysis, Incremental Cost-Effectiveness, Screening v. No Screening

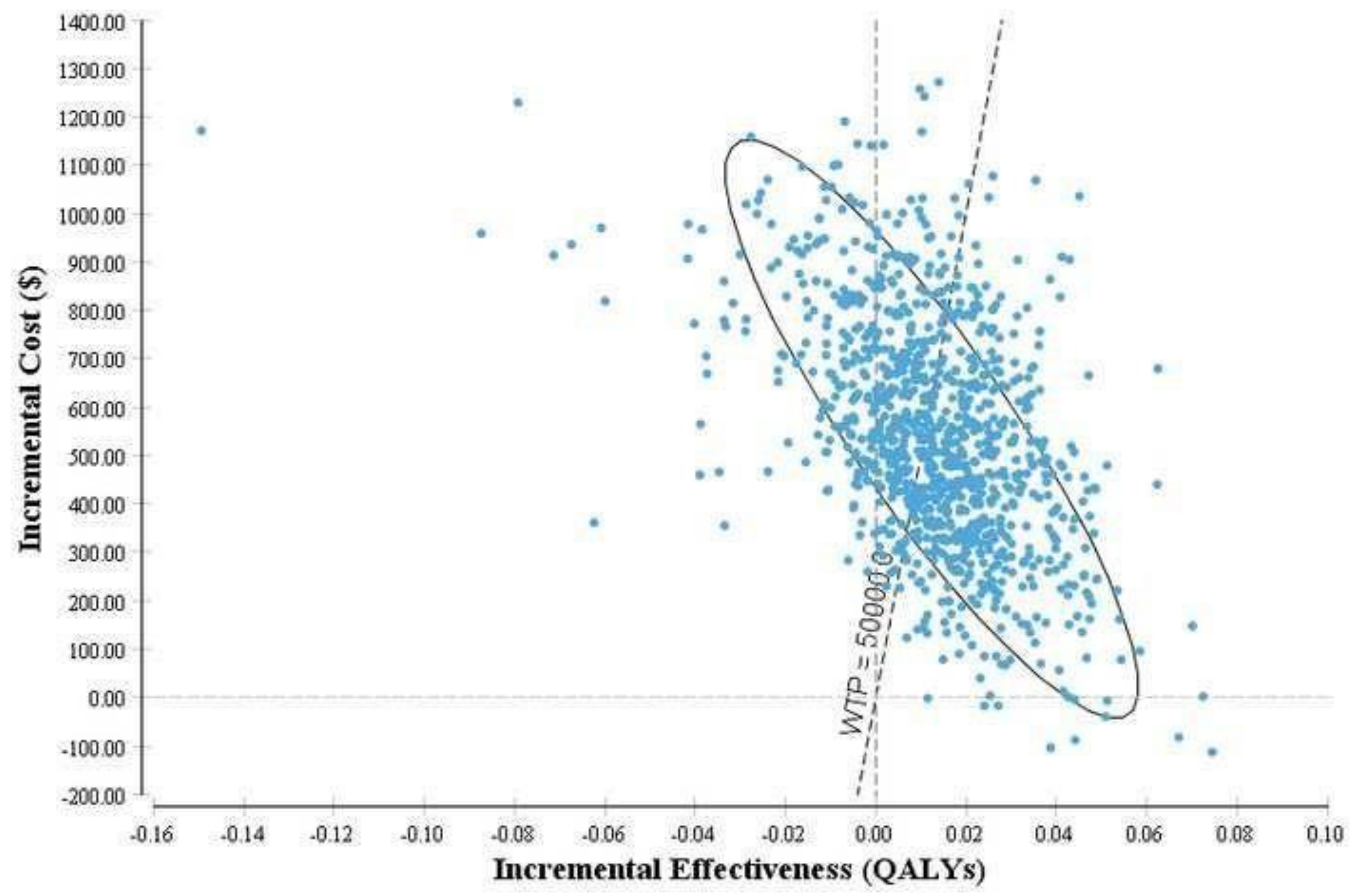




\section{CE Acceptability Curve}

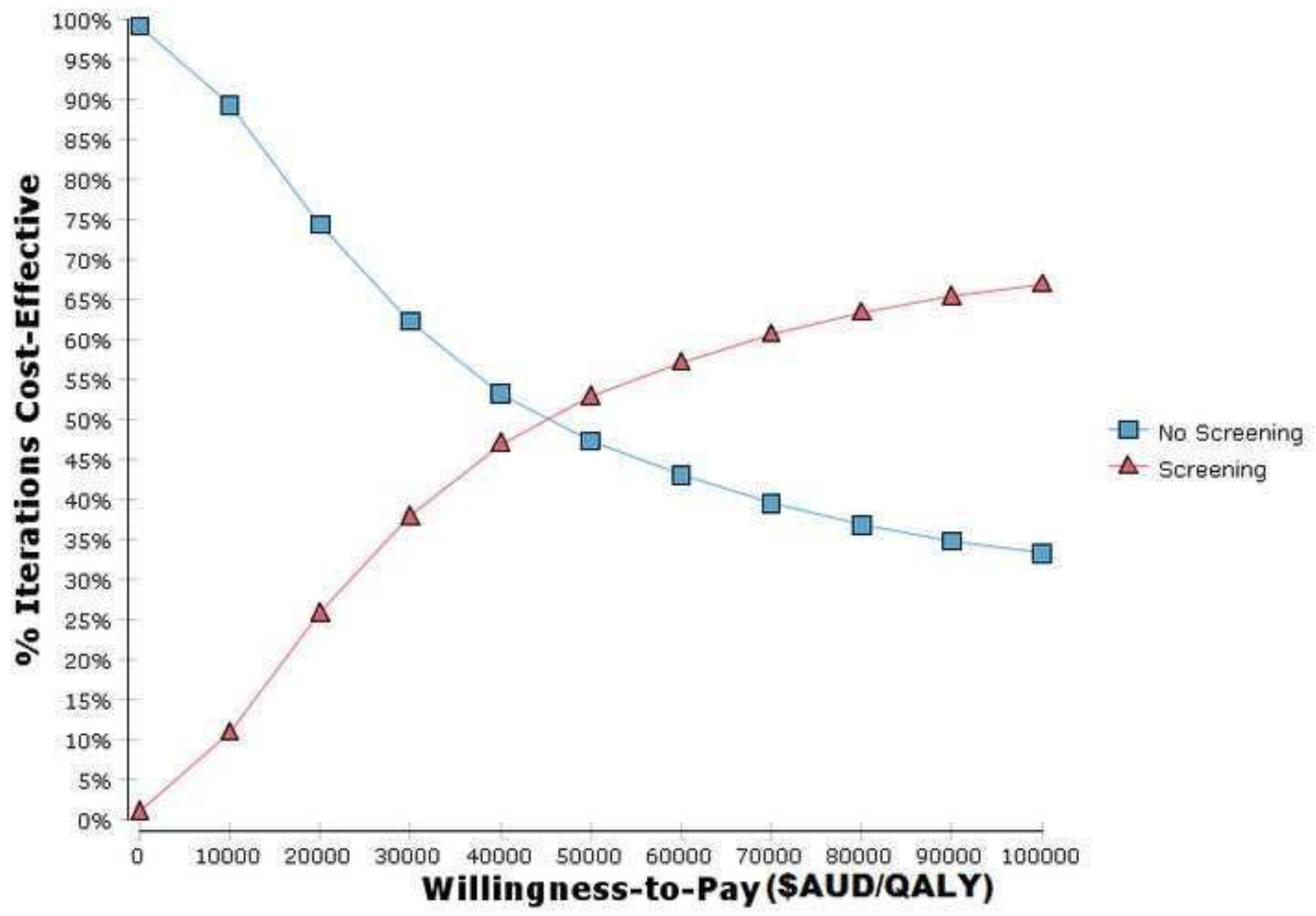

